\title{
Editorial
}

Anaesthesist 2012 - 61:189-190

DOI 10.1007/s00101-012-2004-6

Online publiziert: 18. März 2012

(c) Springer-Verlag 2012

R. Rossaint ${ }^{1}$ B. Z wissler $^{2}$

${ }^{1}$ Klinik für Anästhesiologie, Universitätsklinikum Aachen, Rheinisch-Westfälische

Technische Hochschule Aachen

${ }^{2}$ Klinik für Anästhesiologie, Ludwig-Maximilians-Universität, Klinikum Großhadern, München

\section{Der Anaesthesist}

\section{DIE deutsche Fachzeitschrift für Originalarbeiten}

Mit dem Ausscheiden von Prof. Dr. R. Larsen verjüngte sich Anfang 2010 das Schriftleiterteam unserer Zeitschrift Der Anaesthesist. Nach mehr als 2 Jahren ist es an der Zeit, eine kurze Zwischenbilanz zu ziehen. Primäres Ziel war und ist es, den Lesern Monat für Monat mithilfe aktueller Übersichts- und Weiterbildungsbeiträge klinisch relevante Informationen zu allen Säulen unseres Faches auf höchstem Niveau anzubieten. Gleichzeitig sollte Der Anaesthesist jedoch auch als das deutschsprachige Forum für hochwertige Originalarbeiten und Kasuistiken in unserem Fach weiter ausgebaut werden. Dies ist kein geringer Anspruch in einer Zeit, in der der „impact factor“ - sowohl für Autoren als auch für Kliniken und Fakultäten - zur entscheidenden Wissenschaftswährung geworden ist, und es daher zunehmend schwieriger wird, gute Originalbeiträge zu erhalten. Um in dem hochkompetitiven Umfeld der etablierten sowie v. a. auch der neuen, rein elektronischen Zeitschriften, die inzwischen sehr aktiv um Autoren werben, bestehen zu können, haben die Schriftleitung und das Herausgebergremium von Der Anaesthesist 2010 drei wichtige Än- derungen für Originalbeiträge und $\mathrm{Ka}$ suistiken beschlossen [1]:

- Einführung des „editorial manager“ (EM): Der EM erlaubt es, alle Prozessschritte von der Einreichung einer Arbeit über die verschiedenen Phasen der Begutachtung bis zu ihrer Publikation (oder Ablehnung) vollelektronisch durchzuführen. Damit verbunden waren - neben der Einsparung von Papier - zwei wesentliche Ziele:

- Schaffung maximaler Transparenz: Der Publikationsprozess sollte für die Autoren und Gutachter zu jedem Zeitpunkt einsehbar sowie nachvollziehbar sein.

- Erhöhung der Prozessgeschwindigkeit: Schnelle Bearbeitungszeiten scheinen bei den Autoren ein wesentliches Kriterium für die Auswahl einer Fachzeitschrift zur Publikation ihrer Arbeiten zu sein. Ziel war es daher, die initiale Begutachtungszeit, d. h. die Zeit zwischen der Manuskripteinreichung und dem ersten Bescheid (einschließlich Übersendung der Gutachten) auf $<5$ Wochen zu reduzieren.

Tab. 1 Statistik der positiv begutachteten Originalarbeiten und Kasuistiken im Zeitraum 1.2. 2010 bis 31.1.2012

\begin{tabular}{|c|c|c|c|c|c|c|}
\hline \multirow{2}{*}{$\begin{array}{l}\text { Ak- } \\
\text { zep- } \\
\text { tiert }\end{array}$} & \multicolumn{4}{|c|}{ Dauer zwischen ... } & \multirow{2}{*}{$\begin{array}{l}\text { Beim } \\
\text { Autor }^{\mathrm{a}}\end{array}$} & \multirow{2}{*}{$\begin{array}{l}\text { Bei den } \\
\text { Gutach- } \\
\text { tern }^{b}\end{array}$} \\
\hline & $\begin{array}{l}\text { Einreichung } \\
\text { und finaler } \\
\text { Akzeptanz }\end{array}$ & $\begin{array}{l}\text { Einreichung } \\
\text { und Ableh- } \\
\text { nung }\end{array}$ & $\begin{array}{l}\text { Einreichung } \\
\text { und Einladung } \\
\text { der Gutachter }\end{array}$ & $\begin{array}{l}\text { Einreichung } \\
\text { bis zur ersten } \\
\text { Entscheidung }\end{array}$ & & \\
\hline $51 \%$ & 115 Tage & 39Tage & 4 Tage & 40 Tage & 45 Tage & 53 Tage \\
\hline
\end{tabular}


- Einführung ausführlicherer englischer Abstracts: Diese Maßnahme soll internationale Autoren in die Lage versetzen, die in unserer Zeitschrift publizierten Originalarbeiten besser $\mathrm{zu}$ verstehen und diese ggf. dann auch referenzieren zu können.

- Akzeptanz englischsprachiger Originalarbeiten: Diese Maßnahme sollte es auch nicht deutsch sprechenden Autoren erlauben, gute Arbeiten in der Zeitschrift Der Anaesthesist zu publizieren.

Was haben wir erreicht? In den ersten 24 Monaten nach Einführung des EM im Februar 2010 wurden 130 Originalarbeiten und 71 Kasuistiken eingereicht.

Das Redaktionssystem des EM, das sich international bereits bei unterschiedlichsten Fachzeitschriften bewährt hat, erwies sich auch für Der Anaesthesist als praxistauglich. Lange Postwege von Datenträgern oder Papierversionen sowie aus Sicht der Autoren undurchschaubare Begutachtungsprozesse gehören nun der Vergangenheit an. Der Weg über die Onlineeinreichung der Autoren zu den Editoren und von dort zu den Gutachtern ist unkompliziert, schnell und für alle Beteiligten $\mathrm{zu}$ jedem Zeitpunkt nachvollziehbar. Dieses Angebot wird gern angenommen; aus aller Welt senden Autoren interessante Arbeiten ein. Hiermit wurden die gewünschte Transparenz und Effizienz im Begutachtungsverfahren erreicht (• Tab. 1).

Die für die Autoren bedeutsame zeitnahe Entscheidung über ein eingereichtes Manuskript und auch die - bei positiver Begutachtung - rasche Publikation sind gut gelungen. So wurde in den ersten 2 Jahren bei den eingereichten Manuskripten eine finale Entscheidung im Fall einer Akzeptanz nach 115 Tagen und bei Ablehnung nach 39 Tagen getroffen. Somit wurden die gesetzten Ziele im Hinblick auf die schnelle Bearbeitung nahezu $100 \%$ ig erreicht. An dieser Stelle möchten sich die Schriftleiter nochmals ganz besonders bei den Gutachtern der Beiträge bedanken, die durchschnittlich nur 16 Tage für ihr Gutachten benötigten und damit ganz maßgeblich für das schnelle Begutachtungsverfahren verantwortlich sind.
Auch gelang es, die Zeitschrift für englischsprachige Beiträge interessant zu machen. Von den zahlreichen eingereichten Beiträgen $(n=33)$ musste allerdings ein Großteil aufgrund methodischer Unzulänglichkeiten abgelehnt werden.

Ob es der Zeitschrift langfristig gelingt, einen Impact factor $>1$ zu erreichen, kann aktuell noch nicht abgesehen werden. In der Regel führen Änderungen wie z. B. ausführlichere Abstracts oder qualitativ hochwertige Manuskripte erst nach mehreren Jahren zu einer erhöhten Zitationsrate. Hier hilft natürlich besonders, wenn die deutsch sprechenden Autoren bei Publikationen in englischsprachigen Fachzeitschriften auch die guten Beiträge unserer Zeitschrift zitieren.

\section{Fazit}

Zwei Jahre Arbeit mit dem EM und einer internationaleren Ausrichtung der dennoch weiterhin grundsätzlich deutschsprachigen Fachzeitschrift ließen die Attraktivität von Der Anaesthesist weiter steigen, sodass das Schriftleiterteam mit den Rubrikherausgebern sicher ist, Ihnen, unseren Lesern und Autoren, auch in Zukunft - entsprechend den Vorgaben der Zeitschrift [2] - Publikationen von höchster Qualität zu gewährleisten.

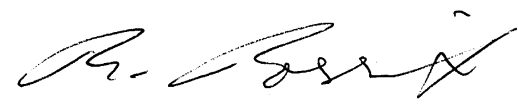

R. Rossaint

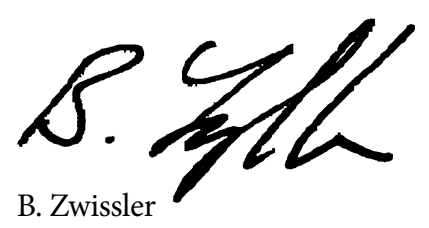

\section{Korrespondenzadressen}

Prof. Dr. R. Rossaint

Klinik für Anästhesiologie, Universitätsklinikum Aachen, Rheinisch-Westfälische Technische Hochschule Aachen

Pauwelsstr. 30, 52074 Aachen

RRossaint@ukaachen.de

Prof. Dr. B. Zwissler

Klinik für Anästhesiologie, Ludwig-MaximiliansUniversität, Klinikum Großhadern

Marchioninistr. 15, 81377 München

Bernhard.Zwissler@med.uni-muenchen.de

\section{Literatur}

1. Zwissler B (2010) Bewährtes und Neues für Der Anaesthesist. Anaesthesist 59:7-8

2. Rossaint R, Zwissler B (2011) Wissenschaftliche Originalarbeiten: Konzeption, Durchführung und Planung. Anaesthesist 60:5-7 